\section{Anemia's Gas6 pedal}

\section{By Linda A. Goldstein, Senior Writer}

A relatively large percentage of anemic patients who receive therapy with erythropoiesis-stimulating agents are either hyporesponsive or resistant to erythropoietin. Researchers at the University Hospital Center and University of Lausanne may have found a new way of treating these patients' anemia using a protein called growth arrestspecific gene 6 , but much work still needs to be done to see if it avoids the problems caused by erythropoiesis-stimulating agents.

Until now, hyporesponsive or resistant patients have typically been treated with higher doses of erythropoiesis-stimulating agents (ESAs), which can raise the risk of mortality and cardiovascular events. Over the past 12 months, an increasing amount of clinical data have shown the problems with this strategy. ${ }^{1-3}$ As a result, the FDA and the Centers for Medicare and Medicaid Services have moved to limit the use of ESAs.

Indeed, in 2007, sales of Amgen Inc.'s

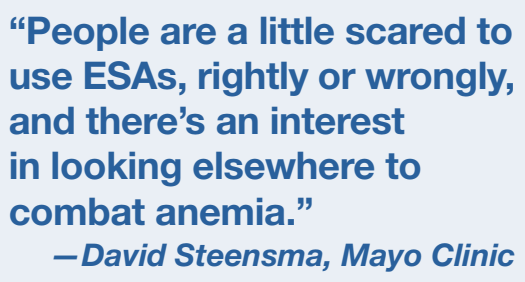

"People are a little scared to use ESAs, rightly or wrongly, and there's an interest in looking elsewhere to combat anemia." -David Steensma, Mayo Clinic

against the development of anemia and facilitated hematocrit recovery.

In additional mouse models of EPO resistance and insufficiency, Gas6 amplified EPO's effect of increasing hematocrit levels after induction of both acute and chronic anemia.

The authors concluded that Gas6 plays a role in erythropoiesis and therefore might have the potential to treat anemic patients who are hyporesponsive or resistant to ESA treatment.

In terms of the predictive value of the results from murine models, "erythropoiesis in mice follows the same rules [as] in humans; most pathways are shared among species, and even single components are quite preserved across evolution," Antonio Risitano, from the Division of Hematology, Federico II University of Naples, told SciBX.

Although primates would be the best animal model, Risitano thinks that mice may be a good surrogate. "In my opinion, the mouse model is very important and may give pivotal information." Even so, Risitano cautioned that "...humans are quite different, and interpretations from the animal models have to be drawn very carefully."

In humans, the erythropoietic response to anemia is induced in the spleen and bone marrow. In mice, the response occurs primarily in the spleen. Nevertheless, Angelillo-Scherrer and colleagues replicated their genetic results in splenectomized animals with induced anemia.

According to Risitano, one of the next steps is to develop a model that will "match [the anemia] affecting humans as a direct consequence of the defect or shared pathogenic pathways. Once you Aranesp darbepoetin alfa fell $12 \%$ compared to 2006 , including a $23 \%$ decline in U.S. sales. U.S. sales of Amgen's Epogen epoetin alpha were down 1\%. Johnson \& Johnson's full-year 2007 earnings showed a $12.3 \%$ drop in worldwide sales of its Procrit/Eprex epoetin, including an $18.2 \%$ slide in the United States.

"People are a little scared to use ESAs, rightly or wrongly, and there's an interest in looking elsewhere to combat anemia," said David Steensma, professor of hematology and oncology at the Mayo Clinic.

Enter growth arrest-specific gene 6 (Gas6). The soluble, vitamin $\mathrm{K}$-dependent protein was discovered by an Italian research group in 1993. ${ }^{4}$ Since then, Gas6 has been studied extensively and shown to be important in the proliferation, survival, migration and adhesion of different cell types in diverse organ tissues.

Most recently, Anne Angelillo-Scherrer and colleagues at the University Hospital Center and University of Lausanne published a paper in the Journal of Clinical Investigation describing the results of a series of preclinical experiments exploring the role of Gas6 in erythropoiesis and anemia. ${ }^{5}$

The key finding is that Gas6 plays a critical role in the generation of erythroid progenitors and erythroblasts, at least in part by enhancing their survival response to erythropoietin (EPO).

In mice lacking Gas6, the erythropoietic response to EPO was reduced, and when anemia was induced in the animals, hematocrit recovery was impaired. By contrast, in wild-type mice, treatment with Gas6 protected have this information, the model may be also suitable to test the potential efficacy of drugs or any other therapeutic strategy."

A key unanswered question is whether Gas6-based therapeutics would circumvent the safety issues associated with the marketed ESAs. According to Steensma, "there is a suggestion that the signaling [of Gas6] overlaps substantially with EPO, so it's not clear that they would be able to get away from the safety concerns that have been raised with EPO."

Research also has shown that Gas6 promotes platelet aggregation and the stabilization of platelet aggregation. "We know that ESAs have a thrombosis risk, so the question is: if you give ESA plus something that increases Gas6, would there be a lot of blood clots?" noted Steensma. "If this moves forward clinically, we'd have to follow it very closely."

Peter Young, VP of research at Affymax Inc., thought the JCI article was "intriguing in that it provides another potential player in how erythropoiesis is driven in mammals. It may play into the hyporesponder side of things, but that would have to be further evaluated in humans. It's an excellent study, but it's in mice, so obviously it's very early in terms of understanding how this molecule plays a role in human anemia."

Affymax's Hematide is in Phase III testing to treat anemia associated with chronic renal failure and in Phase II testing to treat anemia in patients with cancer. The compound is a peptide agonist with an amino acid sequence that is unrelated to naturally occurring EPO. 


\section{TARGETS \& MECHANISMS}

The other late-stage ESA in development is FG-2216 from FibroGen Inc. The small-molecule inhibitor of hypoxia-inducible factor prolyl hydroxylase (HIF-PH) is in Phase II trials to treat anemia in chronic renal failure patients.

\section{REFERENCES}

1. Edelson, S. BioCentury 15(12), A1; March 12, 2007

2. Usdin, S. BioCentury 15(22), A1; May 14, 2007

3. Usdin, S. BioCentury 15(50), A11; Nov. 12, 2007

4. Manfioletti, G. et al. Mol. Cell Biol. 13, 4976-4985 (1993)

5. Angelillo-Scherrer, A. et al. J. Clin. Invest.; published online Jan. 10, 2008; doi: $10.1172 / \mathrm{JCl} 30375$

Contact: Anne Angelillo-Scherrer, University Hospital Center and
University of Lausanne, Lausanne, Switzerland e-mail: Anne.Angelillo-Scherrer@chuv.ch

6. Angelillo-Scherrer, A. et al. J. Clin. Invest. 115, 237-246 (2005)

COMPANIES AND RESEARCH INSTITUTIONS MENTIONED

Affymax Inc. (NASDAQ:AFFY), Palo Alto, Calif.

Amgen Inc. (NASDAQ:AMGN), Thousand Oaks, Calif.

Federico II University of Naples, Naples, Italy

FibroGen Inc., South San Francisco, Calif.

Johnson \& Johnson (NYSE:JNJ), New Brunswick, N.J.

Mayo Clinic, Rochester, Minn.

University Hospital Center, Lausanne, Switzerland

University of Lausanne, Lausanne, Switzerland 